\title{
ULTRASOUND PROMOTED ONE-POT SYNTHESIS OF 2- ARYLIMIDAZO[1,2-A]PYRIMIDINES IN GLYCEROL
}

\author{
Hani Yeslam S. Atif ${ }^{1, \bowtie}$, Devendra S. Wagare ${ }^{2}$, Ahmed Zain Ahmed ${ }^{3}$ \\ and Ayesha N. Durrani ${ }^{1}$ \\ ${ }^{1}$ Department of Chemistry, Dr. Rafiq Zakaria College for Women, Aurangabad (M.S.). India. \\ ${ }^{2}$ Department of Chemistry, Vivekanand Arts, Sardar Dalipsingh Commerce \& Science \\ College, Aurangabad (M.S.). India. \\ ${ }^{3}$ Department of Chemistry, Maulana Azad College Aurangabad, India. \\ ${ }^{\circledR}$ Corresponding Author: hanialsomaity2018@gmail.com
}

\begin{abstract}
In this study, we have designed a simple and highly efficient method for the synthesis of imidazo[1,2-a]pyrimidine derivatives by the cyclo condensation of aromatic ketones with NBS and 2-aminopyrimidine in glycerol as a green reaction medium, by a one-pot procedure and under ultrasound irradiation. In comparison to the methods reported in the literature, this method has various advantages; such as more eco-friendly, clean reaction profile, mild reaction condition, easier work-up procedure, high yield and shorter reaction time. The synthesized compounds were characterized for structural conformation by FT-IR, ${ }^{1} \mathrm{H},{ }^{13} \mathrm{C}$ NMR, and mass spectroscopic techniques.

Keywords: Imidazo[1,2-a]pyrimidines, One-pot, Glycerol, Ultrasound Irradiation.
\end{abstract}

RASĀYAN J. Chem., Vol. 14, No.4, 2021

\section{INTRODUCTION}

For the past few decades, chemists have had an interest in heterocyclic compounds and their derivatives. Heterocyclic compounds have broad application in pharmaceutical and chemical industries. ${ }^{1}$ Imidazofused pyrimidine heterocyclic has great significance in pharmaceutical as well as biomedicals. These scaffolds occur in several natural and biological active molecules. ${ }^{2}$ imidazo[1,2-a]pyrimidine have the considerable interest of chemists in the field of the pharmaceutical industry and these compounds show many biological activities like antimicrobial ${ }^{3}$, anti-fungal ${ }^{4}$, anticancer ${ }^{5}$, anti-inflammatory ${ }^{6}$, antitubercular $^{7}$, antiviral ${ }^{8}$, antibacterial ${ }^{9}$, antipyretic ${ }^{10}$, anti-HIV ${ }^{11}$, with their analgesic. ${ }^{12}$ They also possess an antagonistic activity against purines ${ }^{13}$, GABA receptor ligands ${ }^{14}$, local aesthetic and calcium channel blocking activity ${ }^{15}$, and inhibitor of bone resorption YM 529 was effective in phase III clinical trial to treat osteoporosis. ${ }^{16}$ Furthermore, pyrimidines can be used as blue fluorescent light emitters and photochemical sensors. ${ }^{17}$ It was noticed that Glycerol acts as an "organic water" because of its properties which are similar to water like polarity, easily available, cheap, biodegradability, non-toxic and easily forming hydrogen-bond. It has a broad range of solubility in organic and inorganic compounds together with transition metal catalysts. ${ }^{18}$ Glycerol also shows some peculiar chemical and physical properties such as minimum toxicity, non-volatile solvent, easy removal from a reaction mixture as well as its high boiling point and being prepared effortlessly from renewable feedstocks. ${ }^{19}$ Commonly it is used as a reaction green medium in several organic transformations like cross-coupling reactions ${ }^{20}$ that involved Pd-catalyzed Heck and Suzuki cross-couplings, acid and base-catalyzed condensations, catalytic hydrogenation, and asymmetrical reduction ${ }^{21}$, and then one-pot multi-component reaction. ${ }^{22}$ For the past few decades, such application of ultrasonic irradiation as an alternative source of energy has exerted considerable effort. It contributed greatly to the field of organic synthesis. It has widely been used in various organic transformations to create new possibilities in stimulating new reactions that are difficult to be synthesized by traditional methods. In recent years, the sonochemical approach has been developed 
to include the syntheses of fused heterocyclic compounds like benzofuran ${ }^{23}$, pyrimidopyrans ${ }^{24}$, pyridopyrimidines $^{25}$, pyrazolopyridine ${ }^{26}$, and dihydropyranopyrazole ${ }^{27}$. Due to Imidazo[1,2a]pyrimidine broad applications in pharmaceuticals and material fields, great efforts have been made over the past few decades. Imidazo[1,2-a]pyrimidine heterocycles are tremendously significant scaffolds for being used as a synthetic intermediate for synthesizing complex molecules. Several methods have been developed to synthesize imidazo[1,2-a]pyrimidines from the reaction of 2-aminopyrimidines with the $\alpha$ bromoacetophenones in solvents like $\mathrm{DME}^{28}$ ethanol ${ }^{29}, \mathrm{DMF}^{30}$ and acetone ${ }^{31}$. Unfortunately, there is a need for a long reaction time and the yield is poor. In addition to that, $\alpha$-bromoketones are lachrymatory, not readily available and toxic. Therefore, the development of an efficient, mild and eco-friendly method to synthesis 2-arylimidazo[1,2-a]pyrimidines is currently still important in organic synthesis. Herein, The researcher has developed a one-pot and high efficient method for synthesis of imidazo[1,2-a]pyrimidines from the reaction of in-situ-generated $\alpha$-bromoacetophenones and 2-aminopyrimidine using glycerol as a greener reaction medium with the assist of ultrasound irradiation afforded imidazo[1,2-a]pyrimidine derivatives with high yield (Scheme-1).

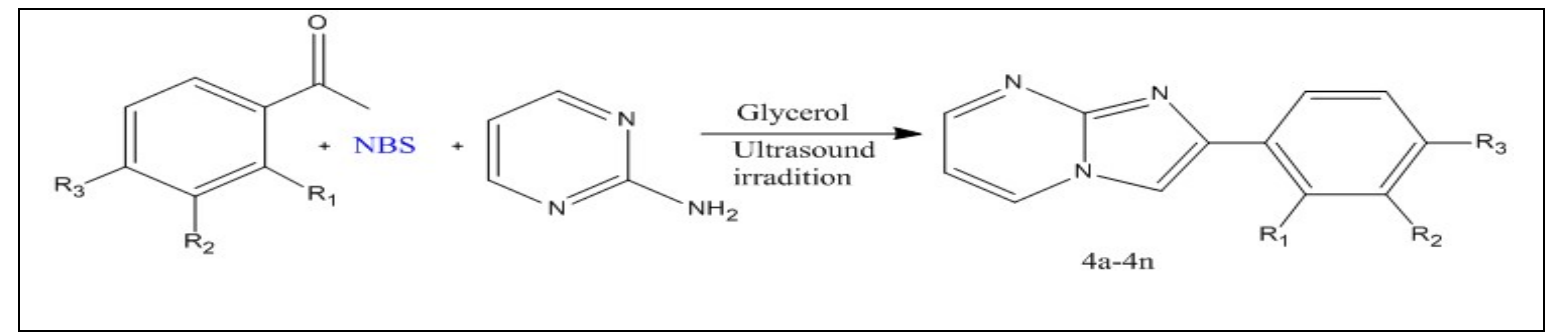

Scheme-1: One-pot Synthesis of imidazo[1,2-a]pyrimidines

\section{Materials and Methods}

\section{EXPERIMENTAL}

All chemicals and solvents used in this study, which are Aldrich chemical companies, were commercially available and purchased from the market. It should be noted that we used these chemicals without any further purification. Melting points were determined in metler melting apparatus and are uncorrected. The ultrasonicator bath is used for irradiation. Formation of the compounds was checked by Thin Layer Chromatography (TLC) on aluminum foil coated s with silica gel 60 F254 plates $0.5 \mathrm{~mm}$ thick. FT-IR spectra were recorded on a Shimadzu FT-IR-8400 spectrometer using the KBr pellet method. Mass spectra were recorded on WATERS, Q-TOF MICROMASS (ESI-MS) by using the direct inlet probe technique. ${ }^{1} \mathrm{H}$ NMR and ${ }^{13} \mathrm{C}$ NMR were recorded in DMSO-d 6 solvent on a Bruker Avance Neo 500-MHz spectrometer.

\section{General Procedure for One-pot Synthesis of 2-Arylimidaz[1,2-a]pyrimidines}

To a mixture of acetophenones $(0.01 \mathrm{~mol})$ and N-bromosuccinimide $(0.01 \mathrm{~mol}), 10 \mathrm{ml}$ of glycerol was added with efficient stirring. The resulting mixture was Sonicated by ultrasound at $80{ }^{\circ} \mathrm{C}$, and the formation of $\alpha$ - bromoacetophenones (intermediate) was checked by Thin Layer Chromatography (TLC). After completion of bromination, to this reaction mixture 2-amino-pyrimidines $(0.01 \mathrm{~mol})$ were added and sonicated at the same reaction condition. After completion of the reaction, the reaction mass was cooled then poured in ice-cold water and neutralized, at $0{ }^{\circ} \mathrm{C}$, with ammonium hydroxide, then extracted with dichloromethane the solid product was obtained and recrystallized with ethanol.

\section{RESULTS AND DISCUSSION}

The imidazo[1,2-a]pyrimidines derivatives seem to have attracted a great deal of interest. Such interest appears to return to their wide application in the field of pharmaceutical and chemistry. Earlier reported methods to synthesize imidazo[1,2-a]pyrimidines have involved refluxing $\alpha$-halo ketones with 2aminopyrimidine in harmful and volatile organic solvents as reaction medium. It is worth noticing that the $\alpha$-halo ketones are unstable, lachrymatric and difficult to deal. ${ }^{32}$ It has prompted the researchers' interest to design an eco-friendly protocol for synthesizing imidazo[1,2-a]pyrimidines in glycerol as a green 
RASĀYAN J. Chem.

Vol. 14 | No. 4 |2645-2651| October- December | 2021

reaction medium with the assist of ultrasound irradiation. Therefore, the researchers have synthesized insitu $\alpha$-halo ketones (intermediate) that are followed by a reaction with 2 -aminopyrimidine in the same pot. To find out a better solvent, such a reaction has been examined by using different solvents namely isopropyl alcohol, acetone, ethanol, DMF, pyridine and glycerol. Results are summed up in (Table-1). The researchers have observed that despite the reaction having been completed in all of those solvents; glycerol was the best one affording $88-94 \%$ of the desired product and a shorter reaction time (entry 6). As glycerol seems to be a better solvent for further chemical reactions understudying, the researchers have optimized reaction conditions by using different solvents (Table-1). It has been seen that yield is increased by using glycerol and the reaction time is significantly reduced $(1.10-1.30 \mathrm{hrs})$, by ultrasound irradiation at $80{ }^{\circ} \mathrm{C}$. This glycerol can typically be recovered by extracting the first product and the recovered solvent is can be reused without loss of activity. The results are summarized in (Table-2).

With optimal reaction conditions at hand, Researchers have investigated the scope of this coupling. Such efforts aim to show the generality of this method that was given in the report. Conversion is generally high with a variety of electron-withdrawing (EWG) and electron-donating (EDG) groups, a wide range of acetophenones bearing electron-withdrawing, electron-donating, and electron-neutral groups could be used as coupling partners with NBS and 2-aminopyrimidine which were smoothly transformed to the corresponding 2-Arylimidazo[1,2-a]pyrimidines with excellent yields (Table-2, entries 4a-4n). All meta-, ortho-, and para-substituted ketones are easily converted into the required products. This indicates that steric bulk has probably no significant effect on reactivity.

Table-1: Optimization of Reaction Medium

\begin{tabular}{l|l|l|l|l}
\hline Entry & Solvents & Time $(\mathrm{hrs})$ & Volume $(\mathrm{ml})$ & Yield \% \\
\hline 1 & Isopropyl alcohol & $1.45-2.15$ & 10 & $60-65$ \\
2 & acetone & $2.30-2.50$ & 10 & $55-65$ \\
3 & ethanol & $2.25-2.45$ & 10 & $50-60$ \\
4 & DMF & $1.50-2.10$ & 10 & $65-70$ \\
5 & Pyridine & $2.15-2.40$ & 10 & $60-70$ \\
6 & Glycerol & $1.10-1.30$ & 10 & $88-94$ \\
\hline
\end{tabular}

Reaction conducted through using acetophenone $(0.01 \mathrm{~mol}), \mathrm{NBS}(0.01 \mathrm{~mol})$ and $(0.01 \mathrm{~mol})$ of 2-aminopyrimidines.

Table-2: Synthesis of imidazo[1,2-a]pyrimidines (4a-4n)

\begin{tabular}{c|c|c|c|c|c|c}
\hline Product & $\mathrm{R}$ & $\mathrm{R}_{1}$ & $\mathrm{R}_{2}$ & Reaction time(hrs) $^{\mathrm{b}}$ & Melting point(M.p) & ${\text { Yield } \%^{\mathrm{a}}}$ \\
\hline $4 \mathrm{a}$ & $\mathrm{H}$ & $\mathrm{H}$ & $\mathrm{H}$ & 1.25 & $196-198$ & 90 \\
\hline $4 \mathrm{~b}$ & $\mathrm{H}$ & $\mathrm{H}$ & $4-\mathrm{CH}_{3}$ & 1.22 & $228-230$ & 92 \\
\hline $4 \mathrm{c}$ & $\mathrm{H}$ & $\mathrm{H}$ & $4-\mathrm{Cl}$ & 1.10 & $272-274$ & 94 \\
\hline $4 \mathrm{~d}$ & $\mathrm{H}$ & $3-\mathrm{Cl}$ & $\mathrm{H}$ & 1.27 & $193-195$ & 89 \\
\hline $4 \mathrm{e}$ & $2-\mathrm{Cl}$ & $\mathrm{H}$ & $4-\mathrm{Cl}$ & 1.18 & $250-252$ & 91 \\
\hline $4 \mathrm{f}$ & $\mathrm{H}$ & $3-\mathrm{OCH}_{3}$ & $\mathrm{H}$ & 1.30 & $222-224$ & 90 \\
\hline $4 \mathrm{~g}$ & $\mathrm{H}$ & $3-\mathrm{NO}_{2}$ & $\mathrm{H}$ & 1.28 & $244-246$ & 89 \\
\hline $4 \mathrm{~h}$ & $\mathrm{H}$ & $\mathrm{H}$ & $4-\mathrm{OCH}_{3}$ & 1.17 & $190-192$ & 88 \\
\hline $4 \mathrm{i}$ & $\mathrm{H}$ & $\mathrm{H}$ & $4-\mathrm{NO}_{2}$ & 1.15 & $364-368$ & 90 \\
\hline $4 \mathrm{j}$ & $\mathrm{H}$ & $\mathrm{H}$ & $4-\mathrm{F}$ & 1.12 & $226-228$ & 92 \\
\hline $4 \mathrm{k}$ & $\mathrm{H}$ & $3-\mathrm{OCH}$ & $4-\mathrm{OCH}$ & 1.30 & $154-156$ & 89 \\
\hline $4 \mathrm{l}$ & $\mathrm{H}$ & $3-\mathrm{Cl}$ & $4-\mathrm{Cl}$ & 1.20 & $223-225$ & 90 \\
\hline $4 \mathrm{~m}$ & $2-\mathrm{CH}$ & $\mathrm{H}$ & $\mathrm{H}$ & 1.26 & $260-263$ & 88 \\
\hline $4 \mathrm{n}$ & $\mathrm{H}$ & $\mathrm{H}$ & $4-\mathrm{Br}$ & 1.10 & $212-114$ & 94 \\
\hline
\end{tabular}

${ }^{\mathrm{a}}$ Isolated yield. High yields and shorter reaction time in green medium are remarkable features.

${ }^{\mathrm{b}}$ Time for overall reaction

The prepared compounds were characterized for structural conformation by $\mathrm{FT}-\mathrm{IR},{ }^{1} \mathrm{H},{ }^{13} \mathrm{C}$ NMR, and mass spectroscopic techniques. The FT-IR spectra of compounds $4 \mathrm{a}-\mathrm{n}$ showed there is no peak for $-\mathrm{NH}_{2}$, in the 3195-3290 region and there is no peak for the Carbonyl carbon group in the region $1785-1835 \mathrm{~cm}^{-1}$. Also, the FT-IR spectra of compounds 4a-n exhibited average bands in the range $1646-1515 \mathrm{~cm}^{-1}$ (aromatic carbons and $\mathrm{C}=\mathrm{N}$ stretching). The ${ }^{1} \mathrm{H}-\mathrm{NMR}$ data of compounds $4 \mathrm{a}-\mathrm{n}$ showed there is a single peak at around $\delta(7.58-8.86 \mathrm{ppm})$ belonging to the hydrogen of the imidazole ring. ${ }^{13} \mathrm{C}-\mathrm{NMR}$ data of 
RASĀYAN J. Chem.

Vol. 14 | No. 4 |2645-2651| October- December | 2021

compounds 4a-n showed there is no peak belonging to the carbonyl carbon of the acetophenone group around $192 \mathrm{ppm}$, but appeared signals only for aromatic carbons in the range 107-160, aside from the signal of $\mathrm{CH}_{3}$ group around $20.77 \mathrm{ppm}$ and signals of $\mathrm{OCH}_{3}$ group around (55-56 ppm). This gives sturdy evidence that the carbonyl carbon group of haloketone is converted to alkene ${ }^{33}$. The mechanism of this method is shown in (Fig.-1). In the presence of glycerol, NBS Initially, came out of bromine as a cation and the acetophenones as enol form attacked on bromine to generate $\alpha$-bromoacetophenones (intermediate). The prime part of the mechanism includes elementary coupling reactions between $\alpha$ bromoacetophenones and endocyclic nitrogen of 2-aminopyrimidine and followed by a cyclization reaction to form imidazo[1,2-a]pyrimidines.

\section{Characterization data of imidazo[1,2-a]Pyrimidines [4a-4n] \\ 2-Phenylimidazo[1,2-a]pyrimidine (4a)}

Mp 196-198 ${ }^{0} \mathrm{C} ;{ }^{1} \mathrm{H}$ NMR $\left(500 \mathrm{MHz}, \mathrm{DMSO}-\mathrm{d}_{6}\right): \delta 8.53(1 \mathrm{H}, \mathrm{d}, J=6.61 \mathrm{~Hz}), 7.82(1 \mathrm{H}, \mathrm{s}), 7.49(1 \mathrm{H}, \mathrm{dd}$, $J=1.70 \mathrm{~Hz}, J=4.34 \mathrm{~Hz}), 7.29(2 \mathrm{H}, \mathrm{d}, J=6.42 \mathrm{~Hz}), 7.18(1 \mathrm{H}, \mathrm{dd}, J=4.34 \mathrm{~Hz}, J=6.61 \mathrm{~Hz}), 7.14-7.16$ $(3 \mathrm{H}, \mathrm{m}) ;{ }^{13} \mathrm{C}$ NMR $\left(500 \mathrm{MHz}, \mathrm{DMSO}_{6}\right) \delta 153.14,149.12,139.04,133.60,129.14,128.94,127.16$, 125.93, 108.52, 107.93; IR (KBr) $\mathrm{cm}^{-1}:$ 1646, 1524, 1078, 771; ESI-MS (m/z): 196 [M+H]+.

\section{2-(p-Tolyl)imidazo[1,2-a]pyrimidine (4b)}

Mp 228-230 ${ }^{0} \mathrm{C} ;{ }^{1} \mathrm{H}$ NMR $\left(500 \mathrm{MHz}, \mathrm{DMSO}-\mathrm{d}_{6}\right): \delta 8.87-8.88(1 \mathrm{H}, \mathrm{dd}, J=4.2 \mathrm{~Hz}, J=2.1 \mathrm{~Hz}), 8.44-8.45$ $(1 \mathrm{H}, \mathrm{dd}, J=6.8 \mathrm{~Hz}, J=2.1 \mathrm{~Hz}), 8.25(1 \mathrm{H}, \mathrm{s}), 7.81-7.83(2 \mathrm{H}, \mathrm{d}, J=8.1, \mathrm{~Hz}), 7.19-7.21(2 \mathrm{H}, \mathrm{d}, J=8.1$ $\mathrm{Hz}), 6.96-6.98(1 \mathrm{H}, \mathrm{dd}, J=6.9 \mathrm{~Hz}, J=4.2 \mathrm{~Hz}), 2.27\left(3 \mathrm{H}, \mathrm{s}, \mathrm{CH}_{3}\right) ;{ }^{13} \mathrm{C}$ NMR $\left(500 \mathrm{MHz}, \mathrm{DMSO}-\mathrm{d}_{6}\right): \delta$ $150.00,147.82,145.17,137.55,134.79,130.39,129.25$ (2C), 125.56 (2C), 108.74, 106.96, 20.77; IR (KBr) $\mathrm{cm}^{-1} 3127,1614,1512$; ESI-MS: $(\mathrm{m} / \mathrm{z}) 210.65[\mathrm{M}+\mathrm{H}]+$.

2-(4-chlorophenyl)-imidazo[1,2-a]pyrimidine(4c).

Mp 272-274 ${ }^{0} \mathrm{C} ;{ }^{1} \mathrm{H}$ NMR (500 MHz, DMSO-d $\left.\mathrm{d}_{6}\right): \delta 8.95-8.97(1 \mathrm{H}, \mathrm{m}), 8.53-8.55(1 \mathrm{H}, \mathrm{m}), 8.41(1 \mathrm{H}, \mathrm{s})$, 8.01-8.04 (2H, dd, $J=8.4,1.4, \mathrm{~Hz}), 7.52-7.54(2 \mathrm{H}, \mathrm{dd}, J=7.9,1.3 \mathrm{~Hz}), 7.05-7.08(1 \mathrm{H}, \mathrm{m}) ;{ }^{13} \mathrm{C} \mathrm{NMR}$ $\left(500 \mathrm{MHz}, \mathrm{DMSO}_{-} \mathrm{d}_{6}\right) \delta 150.56,147.96,143.93,135.05,132.58,132.23,128.74(2 \mathrm{C}), 127.31(2 \mathrm{C})$, 108.93, 107.82; IR (KBr) cm $\mathrm{cm}^{-1}: 1613,1078,738,678 ;$ ESI-MS (m/z): $230[\mathrm{M}+\mathrm{H}]+$.

\section{2-(3-chlorophenyl)-imidazo[1,2-a]pyrimidine(4d)}

Mp 193-195 ${ }^{0} \mathrm{C} ;{ }^{1} \mathrm{H}$ NMR (500 MHz, DMSO-d 6 ): $\delta 8.97(1 \mathrm{H}, \mathrm{dd}, J 5.7=1.8 \mathrm{~Hz}), 8.58(1 \mathrm{H}, \mathrm{dd}), 8.52$ $(1 \mathrm{H}, \mathrm{s}), 8.23(1 \mathrm{H}, \mathrm{s}), 7.97(1 \mathrm{H}, \mathrm{d}), 7.52(1 \mathrm{H}, \mathrm{t}, J 4.5=8.0 \mathrm{~Hz}, J 5.7=7.7 \mathrm{~Hz}), 7.43(1 \mathrm{H}, \mathrm{d}, J=8.2 \mathrm{~Hz})$, $7.07(1 \mathrm{H}, \mathrm{dd}, J 5.5=6.7 \mathrm{~Hz}, J 6.7=4.0 \mathrm{~Hz}) ;{ }^{13} \mathrm{C}$ NMR $\left(500 \mathrm{MHz}, \mathrm{DMSO}-\mathrm{d}_{6}\right) \delta 154.32,147.64,139,87$, 135.63, 134.98, 134.34, 129.82, 129.32, 127.61, 125.32, 109.74, 107.23; IR (KBr) cm ${ }^{-1}: 1616,1508$, 1087, 789; ESI-MS (m/z) $230[\mathrm{M}+\mathrm{H}]^{+}$.

\section{2-(2,4-dichlorophenyl)-imidazo[1,2-a]pyrimidine(4e)}

Mp 250-252 ${ }^{\circ} \mathrm{C}^{1} \mathrm{H}$ NMR(500 MHz, DMSO-d $\left.{ }_{6}\right): \delta 9.12(1 \mathrm{H}, \mathrm{dd}, J=2.13 \mathrm{~Hz}, J=6.71 \mathrm{~Hz}), 8.84(1 \mathrm{H}, \mathrm{dd}$, $J=1.98 \mathrm{~Hz}, J=4.27 \mathrm{~Hz}), 8.63(1 \mathrm{H}, \mathrm{s}), 7.67(2 \mathrm{H}, \mathrm{dd}, J=1.98 \mathrm{~Hz}, J=8.24 \mathrm{~Hz}), 7.42(1 \mathrm{H}, \mathrm{dd}, J=1.98$ $\mathrm{Hz}, J=8.24 \mathrm{~Hz}), 7.28(1 \mathrm{H}, \mathrm{dd}, J=4.27 \mathrm{~Hz}, J=6.71 \mathrm{~Hz}) ;{ }^{13} \mathrm{C}$ NMR $\left(500 \mathrm{MHz}, \mathrm{DMSO}-\mathrm{d}_{6}\right) \delta 154.74$, $148.43,140.22$, 135.87, 135.34, 132.98, 131.12, 130.43, 128.13, 126.76, 109.33, 107,54; IR (KBr) cm ${ }^{-1}$ : 1612, 1500, 1045, 710 ; ESI-MS: $(\mathrm{m} / \mathrm{z}) 263[\mathrm{M}+\mathrm{H}]+$.

\section{2-(3-methoxyphenyl)imidazo[1,2-a]pyrimidine(4f)}

Mp 221-223 ${ }^{0} \mathrm{C} ;{ }^{1} \mathrm{H}$ NMR (500 MHz, DMSO-d 6 ): $\delta 8.53(1 \mathrm{H}, \mathrm{dd}, J=4.3 \mathrm{~Hz}, J=2.1 \mathrm{~Hz}), 8.42(1 \mathrm{H}, \mathrm{dd}, J$ $=6.5 \mathrm{~Hz}, J=2.0 \mathrm{~Hz}), 8.27(2 \mathrm{H}, \mathrm{m}), 7.80(1 \mathrm{H}, \mathrm{s}), 7.38(2 \mathrm{H}, \mathrm{m}), 6.84(1 \mathrm{H}, \mathrm{m}), 3.74\left(3 \mathrm{H}, \mathrm{s}, \mathrm{OCH}_{3}\right) .{ }^{13} \mathrm{C}$ NMR (500 MHz, DMSO-d 6 ) $\delta 160.14,153.61,149.35,140.42,134.26,133.72,129.64,119.22,115.11$, 114.42, 109.17, 107.22, 55.62; IR (KBr) $\mathrm{cm}^{-1}: 3129,1613,1517$; ESI-MS (m/z): $225[\mathrm{M}+\mathrm{H}]+$.

\section{2-(3-nitrophenyl)limidazo[1,2-a]pyrimidine $(4 \mathrm{~g})$}

Mp 244-246 ${ }^{0} \mathrm{C} ;{ }^{1} \mathrm{H}$ NMR $(500 \mathrm{MHz}$, DMSO-d 6 ): $\delta 8.74-8.78(1 \mathrm{H}, \mathrm{dd}, J=3.9 \mathrm{~Hz}, J=2.1 \mathrm{~Hz}), 8.52-8.56$ $(1 \mathrm{H}, \mathrm{dd}, J=6.6 \mathrm{~Hz}, J=2.1 \mathrm{~Hz}), 8.38-8.43(2 \mathrm{H}, \mathrm{m}), 8.13-8.19(1 \mathrm{H}, \mathrm{m}), 7.93(1 \mathrm{H}, \mathrm{s}), 7.58-7.61(1 \mathrm{H}, \mathrm{m})$, 


\section{RASĀYAN J. Chem.}

Vol. 14 | No. 4 |2645-2651| October- December | 2021

6.83-6.87 $(1 \mathrm{H}, \mathrm{dd}, J=6.6 \mathrm{~Hz}, J=4.2 \mathrm{~Hz}) ;{ }^{13} \mathrm{C}$ NMR (500 MHz, DMSO-d 6 ): $\delta 150.83,148.82,147.87$, $146.10,133.34,132.17,129.83,129.47,123.10,120.78,109.37,107.15 ;$ IR $(\mathrm{KBr}) \mathrm{cm}^{-1} 3075,1580$, 1509, 1339; ESI-MS (m/z): $240.71[\mathrm{M}+\mathrm{H}]+$.

\section{2-(4-Methoxyphenyl)-imidazo[1,2-a]pyrimidine(4h)}

Mp 190-192 ${ }^{0} \mathrm{C} ;{ }^{1} \mathrm{H}$ NMR (500 MHz, DMSO-d 6 ): $\delta 8.40-8.43(1 \mathrm{H}, \mathrm{dd}, J=3.9 \mathrm{~Hz}, J=1.8 \mathrm{~Hz}), 8.33-8.36$ $(1 \mathrm{H}, \mathrm{dd}, J=6.6 \mathrm{~Hz}, J=1.8 \mathrm{~Hz}), 7.91-7.96(\mathrm{~d}, J=8.7 \mathrm{~Hz}, 2 \mathrm{H}), 7.58(\mathrm{~s}, 1 \mathrm{H}), 6.87-6.93(\mathrm{~d}, J=8.6 \mathrm{~Hz}, 2 \mathrm{H})$, 6.76-6.78 $(1 \mathrm{H}, \mathrm{dd}, J=6.6 \mathrm{~Hz}, J=4.2 \mathrm{~Hz}), 3.75\left(3 \mathrm{H}, \mathrm{s}, \mathrm{OCH}_{3}\right) ;{ }^{13} \mathrm{C}$ NMR $\left(500 \mathrm{MHz}, \mathrm{DMSO}-\mathrm{d}_{6}\right) \delta$ $160.13,149.71,149.35,145.42,133.16,128.12,124.54,114.66,109.16,107.31,55.41$; IR $(\mathrm{KBr}) \mathrm{cm}^{-1}$ : 3134, 1612, 1518; ESI-MS (m/z):225 [M+H]+.

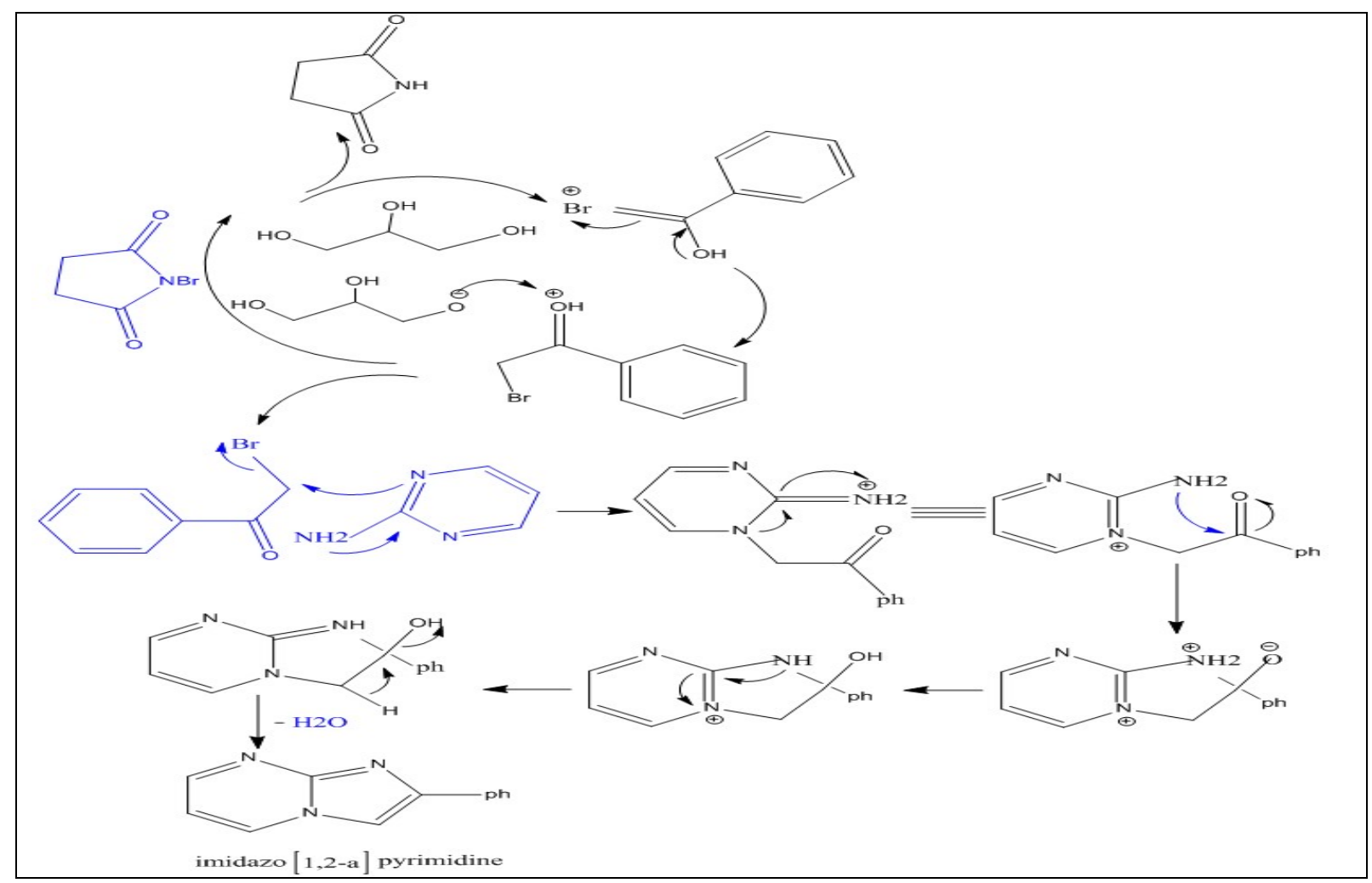

Fig.-1: Mechanism of One-pot Synthesis of imidazo[1,2-a]pyrimidines.

\section{2-(4-Nitrophenyl)-imidazo[1,2-a]pyrimidine(4i)}

Mp 364-368 ${ }^{0} \mathrm{C} ;{ }^{1} \mathrm{H}$ NMR (500 MHz, DMSO-d 6$): \delta 8.77-8.81(1 \mathrm{H}, \mathrm{m}), 8.53-8.57(1 \mathrm{H}, \mathrm{m}), 7.89(1 \mathrm{H}, \mathrm{s})$, 7.53-7.63 (4H, m), 6.86-6.92 (1H, m); ${ }^{13} \mathrm{C}$ NMR (500 MHz, DMSO-d 6 ) $\delta 154.13,149.14,148.32,140.74$, 139.75, 135.20, 126.18, 124.98, 109.54, 107.53; IR (KBr) cm${ }^{-1}: 1591,1511,1343 ;$ ESI-MS (m/z): 240 $[\mathrm{M}+\mathrm{H}]+$.

\section{2-(4-Fluorophenyl)-imidazo[1,2-a]pyrimidine(4j)}

Mp 225-227 ${ }^{0} \mathrm{C} ;{ }^{1} \mathrm{H}$ NMR (500 MHz, DMSO-d $)$ ): $\delta$ 8.53-8.54 $(1 \mathrm{H}, \mathrm{m}), 8.42-8.44(1 \mathrm{H}, \mathrm{m}), 7.99-8.03$ $(2 \mathrm{H}, \mathrm{m}), 7.78(1 \mathrm{H}, \mathrm{s}), 7.12-7.16(2 \mathrm{H}, \mathrm{t}, J=8.7 \mathrm{~Hz}), 6.86-6.89(1 \mathrm{H}, \mathrm{m}) ;{ }^{13} \mathrm{C}$ NMR $\left(500 \mathrm{MHz}, \mathrm{DMSO}-\mathrm{d}_{6}\right)$ $\delta 153.12,148.61,139.56,135.34,134.86,132.86,130.68,127.67,109.97,108.54 ; \mathrm{IR}(\mathrm{KBr}) \mathrm{cm}^{-1}: 1642$, 1097, 757; ESI-MS (m/z): 214 [M+H]+.

\section{2-(3,4-Dimethoxyphenyl)-imidazo[1,2-a]pyrimidine(4k)}

Mp 154-156 ${ }^{0} \mathrm{C}$; ${ }^{1} \mathrm{H}$ NMR (500 MHz, DMSO-d 6 ): $\delta$ 9.04-9.07 $(1 \mathrm{H}, \mathrm{m}), 8.55-8.56(1 \mathrm{H}, \mathrm{m}), 8.52(1 \mathrm{H}, \mathrm{s})$, 7.68-7.71 $(3 \mathrm{H}, \mathrm{m}), 7.12-7.14(1 \mathrm{H}, \mathrm{m}), 3.88(3 \mathrm{H}, \mathrm{s}), 3.84(3 \mathrm{H}, \mathrm{s}) ;{ }^{13} \mathrm{C}$ NMR $\left(500 \mathrm{MHz}, \mathrm{DMSO}-\mathrm{d}_{6}\right) \delta$ 154.87, 152.83, 150.75, 138.63, 133.86, 127.23, 122.01, 112.08, 110.36, 110.24, 109.03, 107.76, 56.14, 55.98; IR (KBr) cm ${ }^{-1}: 1638,1084,728,667$; ESI-MS $(\mathrm{m} / \mathrm{z}): 256[\mathrm{M}+\mathrm{H}]+$. 
RASĀYAN J. Chem.

Vol. 14 | No. 4 |2645-2651| October- December | 2021

\section{2-(3,4-Dichlorophenyl)-imidazo[1,2-a]pyrimidine(4I)}

Mp 223-225 ${ }^{0} \mathrm{C}$; ${ }^{1} \mathrm{H}$ NMR (500 MHz, DMSO-d $)$ : $\delta$ 8.62-8.67 $(1 \mathrm{H}, \mathrm{m}), 8.54-8.56(1 \mathrm{H}, \mathrm{m}), 8.12(1 \mathrm{H}, \mathrm{s})$, 7.65-7.67 (3H, m), 6.88-6.91 (1H, m); ${ }^{13} \mathrm{C}$ NMR (500 MHz, DMSO-d 6 ) $\delta 151.78,148.32,138.16,134.54$, 133.83, 133.41, 133.01, 130.34, 128.43, 127.73, 108.98, 107.92; IR (KBr) cm $\mathrm{cm}^{-1}: 1654,1087,732,668$; ESI-MS $(m / z): 264[\mathrm{M}+\mathrm{H}]+$.

\section{2-(o-Tolyl)imidazo[1,2-a]pyrimidine $(4 \mathrm{~m})$}

Mp 260-263 ${ }^{0} \mathrm{C}$; ${ }^{1} \mathrm{H}$ NMR $\left(500 \mathrm{MHz}, \mathrm{DMSO}_{-}\right)_{6} \delta \mathrm{ppm} 9.24(1 \mathrm{H}, \mathrm{dd}, J=1.70,6.79 \mathrm{~Hz}), 8.98-9.08(1 \mathrm{H}$, $\mathrm{m}), 8.87(1 \mathrm{H}, \mathrm{s}), 7.58-7.72(2 \mathrm{H}, \mathrm{m}), 7.36-7.54(\mathrm{~m}, 3 \mathrm{H}), 2.53(3 \mathrm{H}, \mathrm{s}) ;{ }^{13} \mathrm{C}$ NMR $\left(500 \mathrm{MHz}, \mathrm{DMSO}-\mathrm{d}_{6}\right) \delta$ 153.23, 147.84, 141.22, 135.71, 134.42, 129.53, 129.22, 127.92, 125.41, 122.53, 109.34, 107.11, 19.12; IR (KBr) cm ${ }^{-1}: 3124,1613,1514$; ESI-MS: $m / z 210$ [M+H]+.

\section{2-(4-bromophenyl)-imidazo[1,2-a]pyrimidine(4n)}

Mp 212-214 ${ }^{0} \mathrm{C}$; ${ }^{1} \mathrm{H}$ NMR $\left(500 \mathrm{MHz}, \mathrm{DMSO}-\mathrm{d}_{6}\right): \delta 8.86(1 \mathrm{H}, \mathrm{dd}, J=6.9 \mathrm{~Hz}, J=2.1 \mathrm{~Hz}), 8.52-8.54(1 \mathrm{H}$, $\mathrm{m}), 7.92(2 \mathrm{H}, \mathrm{d}, J=7.9 \mathrm{~Hz}), 7.58(2 \mathrm{H}, \mathrm{d}, J=8.4 \mathrm{~Hz}), 7.79(1 \mathrm{H}, \mathrm{s}), 6.91-6.96(1 \mathrm{H}, \mathrm{m}) ;{ }^{13} \mathrm{C}$ NMR $(500$ $\left.\mathrm{MHz}_{\text {DMSO-d }}\right) \delta 152.93,149.43,136.89,134.96,134.01,133.45,130.19,125.67,109.83,108.47$; IR $(\mathrm{KBr}) \mathrm{cm}^{-1}:$ 1633, 767, 508; ESI-MS (m/z): $275\left(\mathrm{Br}^{79}\right), 277\left(\mathrm{Br}^{81}\right),[\mathrm{M}+\mathrm{H}]+$.

\section{CONCLUSION}

This study has developed a convenient, milder, and efficient one-pot procedure for the synthesis of imidazo[1,2-a]pyrimidines using glycerol as a green reaction medium assisted by ultrasound irradiation. This new protocol provides attractive characteristics in comparison to the conventional methods, such as shorter reaction time, easy procedure, clean reaction profile, high yield, mild reaction condition, operational simplicity and green aspects such as avoiding poisonous catalyst and volatile organic solvents.

\section{ACKNOWLEDGEMENT}

We express our sincere thanks to the principal of Dr. Rafiq Zakaria College for women for providing us with the facilities required for conducting this research work.

\section{REFERENCES}

1. R. Goel, V. Luxami and K. Paul, Royal Society of Chemistry Advances, 5(99), 81608(2015), https://doi.org/10.1039/C5RA14795F

2. Y. Wang, B. Frett and H.Y. Li, Organic Letters, 16(11), 3016(2014), https://doi.org/10.1021/ol501136e

3. G.R. Revankar, T.R. Matthews and R.K. Robins, Journal of Medicinal Chemistry, 18(12), 1253(1975), https://doi.org/10.1021/jm00246a018

4. Y. Rival, G. Grassy, A. Taudou and R. Ecalle, European Journal of Medicinal Chemistry, 26(1), 13(1991), https://doi.org/10.1016/0223-5234(91)90208-5

5. F. Tufail, S. Singh, M. Saquib, J. Tiwari, J. Singh and J. Singh, Chemistry Select, 2(21), 6082(2017), https://doi.org/10.1002/slct.201700557

6. S. Laneri, A. Sacchia, M. Gallitelli, F. Arena, E. Luraschi, E. Abignente and F. Rossi, European Journal of Medicinal Chemistry, 33(3), 163(1998), https://doi.org/10.1016/S0223-5234(98)80005-6

7. G. C. Moraski, L. D. Markley, P. A. Hipskind, H. Boshoff, S. Cho, S. G. Franzblau and M. J. Miller, ACS Medicinal Chemistry Letters, 2(6), 466(2011), https://doi.org/10.1021/ml200036r

8. A. Gueiffier, M. Lhassani, A. Elhakmaoui, R. Snoeck, G. Andrei, O. Chavignon and M. Witvrouw, Journal of Medicinal Chemistry, 39(14), 2856(1996), https://doi.org/10.1021/jm9507901

9. A. Anaflous, N. Benchat, M. Mimouni, S. Abouricha, T. Ben-Hadda, B. El-Bali and B. Hacht, Letters in Drug Design \& Discovery, 1(3), 224(2004), https://doi.org/10.2174/1570180043398885

10. A. Vidal, M. L. Ferrándiz, A. Ubeda, A. Acero-Alarcón, J. Sepulveda-Arques and M. J. Alcaraz, Inflammation Research, 50(6), 317(2001), https://doi.org/10.1007/PL00000250

11. C. Moog, A. Wick, P. Le Ber, A. Kirn and A. M. Aubertin, Antiviral Research, 24(4), 275(1994), https://doi.org/10.1016/0166-3542(94)90075-2 
RASĀYAN J. Chem.

Vol. 14 | No. 4 |2645-2651| October- December | 2021

12. A. Vidal, M. L. Ferrándiz, A. Ubeda, A. Acero-Alarcón, J. Sepulveda-Arques and M. J. Alcaraz, Inflammation Research, 50(6), 317(2001), https://doi.org/10.1007/PL00000250

13. J. W. Chern, C. C. Lee, Y. C. Liaw, and A. J. Wang, Heterocycles (Sendai), 34(6), 1133(1992).

14. A. C. Humphries, E. Gancia, M. T. Gilligan, S. Goodacre, D. Hallett, K. J. Merchant and S. R. Thomas, Bioorganic \& Medicinal Chemistry Letters, 16(6), 1518(2006), https://doi.org/10.1016/j.bmcl.2005.12.037

15. P. J. Sanfilippo, M. Urbanski, J. B. Press, B. Dubinsky and J. J. B. Moore, Journal of Medicinal Chemistry, 31(11), 2221(1988), https://doi.org/10.1021/jm00119a026

16. S. C. Goodacre, L. J. Street, D. J. Hallett, J. M. Crawforth, S. Kelly, A. P. Owens and P. Ferris, Journal of Medicinal Chemistry, 49(1), 35(2006), https://doi.org/10.1021/jm0510651

17. M. Rawat and D. S. Rawat, Tetrahedron Letters, 59(24), 2341(2018), https://doi.org/10.1016/j.tetlet.2018.05.005

18. C. Jiménez-González, P. Poechlauer, Q. B. Broxterman, B. S. Yang, B. D. Am Ende, J. Baird and S. Yee, Organic Process Research \& Development, 15(4), 900(2011), https://doi.org/10.1021/op100327d

19. H. M. Bachhav, S. B. Bhagat and V. N. Telvekar, Tetrahedron Letters, 52(43), 5697(2011), https://doi.org/10.1016/j.tetlet.2011.08.105

20. L. C. Gonçalves, G. F. Fiss, G. Perin, D. Alves, R. G. Jacob and E. J. Lenardão, Tetrahedron Letters, 51(51), 6772(2010), https://doi.org/10.1016/j.tetlet.2010.10.107

21. A. Wolfson, H. Abu-Zaed and D. Tayor, Organic Communications, 9(1), 9(2016)

22. V. Rajeswar Rao and P. Vijaya Kumar, Synthetic Communications, 36(15), 2157(2006), https://doi.org/10.1080/00397910600637012

23. S. S. Palimkar, V. S. More and K. V. Srinivasan, Ultrasonics sonochemistry, 15(5), 853(2008), https://doi.org/10.1016/j.ultsonch.2007.10.006

24. P. Paengphua and S. Chancharunee, Monatshefte für Chemie-Chemical Monthly, 149(10), 1835(2018), https://doi.org/10.1007/s00706-018-2238-3

25. M. H. Mosslemin and M. R. Nateghi, Ultrasonics Sonochemistry, 17(1), 162(2010), https://doi.org/10.1016/j.ultsonch.2009.07.002

26. M. Nikpassand, M. Mamaghani, F. Shirini and K. Tabatabaeian, Ultrasonics Sonochemistry, 17(2), 301(2010), https://doi.org/10.1016/j.ultsonch.2009.08.001

27. Y. Zou, H. Wu, Y. Hu, H. Liu, X. Zhao, H. Ji and D. Shi, Ultrasonics Sonochemistry, 18(3), 708(2011), https://doi.org/10.1016/j.ultsonch.2010.11.012

28. Y. Rival, G. GRASSY and G. MICHEL, Chemical and Pharmaceutical Bulletin, 40(5), 1170(1992), https://doi.org/10.1248/cpb.40.1170

29. D. C. Mohan, S. N. Rao and S. Adimurthy, The Journal of Organic Chemistry, 78(3), 1266(2013), https://doi.org/10.1021/jo3025303

30. Y. Y. Xie, Synthetic Communications, 35(13), 1741(2005), https://doi.org/10.1081/SCC200063911

31. R. J. Sundberg, D. J. Dahlhausen, G. Manikumar, B. Mavunkel, A. Biswas, V. Srinivasan and p. $\begin{array}{llll}\text { Waid, Journal } & \text { Heterocyclic }\end{array}$ https://doi.org/10.1002/jhet.5570250119

32. Ş. G. Küçükgüzel, I. Küçükgüzel, E. Tatar, S. Rollas, F. Şahin, M. Güllüce and L. Kabasakal, European Journal of Medicinal Chemistry, 42(7), 893(2007), https://doi.org/10.1016/j.ejmech.2006.12.038

33. D. S. Ermolat'ev and E. V. Van der Eycken, The Journal of Organic Chemistry, 73(17), 6691(2008), https://doi.org/10.1021/jo8008758

[RJC-6502/2021] 